\title{
Qualitätssicherung von Gesundheitsinformationen im Internet
}

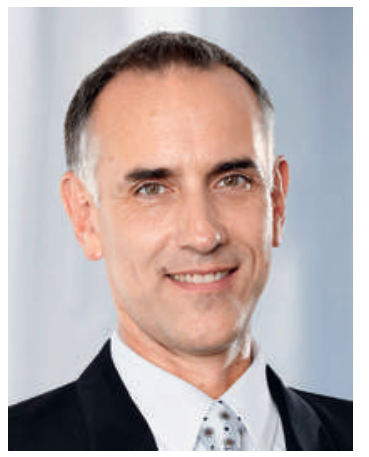

Thema dieses Editorials ist ein zu Unrecht wenig bekannter hochwertiger Service von internationaler Bedeutung im Gesundheitswesen: das Qualitätssicherungslabel Health on the Net, auch als HON bezeichnet. Die 1995 als Stiftung gegründete Non-Governmental-Organisation (NGO) mit Sitz in Genf hat zum Ziel, nützliche, zuverlässige und vertrauenswürdige medizinische Information im Bereich der Gesundheitsversorgung für alle im World Wide Web erkennbar zu machen.

Immer mehr Patienten informieren sich via Internet über Krankheitsbilder und deren Behandlungsmöglichkeiten. Laien fällt es verständlicherweise schwer, zu beurteilen, ob die Informationen sachlich richtig, zuverlässig und aktuell sind. Als Service für die Benutzer ist deshalb die Qualitätssicherung der Inhalte von Websites umso wichtiger. Transparenz ist insbesondere betreffend Herkunft der Informationen und Finanzierung des Angebots notwendig, und die entsprechenden Angaben sollten von einer unabhängigen Stelle geprüft werden.

\section{Das World Wide Web ist für Patienten heute vielfach erste Anlaufstelle bei Gesundheitsfragen.}

Um Informationsquellen im Internet nach formalen Kriterien bezüglich Zuverlässigkeit und Glaubwürdigkeit zu zertifizieren, hat die HON-Stiftung den "HON code of conduct», kurz HONcode, erarbeitet.Gemäss diesem sind acht Prinzipien einzuhalten; unter anderem müssen die Inhalte der Wahrheit entsprechen, dürfen das Arzt-Patienten-Verhältnis nicht stören, und die Vertraulichkeit von individuellen Daten ist zu gewährleisten. Für die Vergabe des entsprechenden Zertifikats werden Inhalte durch die HON-Stiftung auf die Einhaltung der vorgegebenen Kriterien sowohl bei der erstmaligen Zertifizierung als auch nachfolgend in regelmässigen Zeitabständen überprüft. Die Vergabe erfolgt jeweils durch ein neutrales, medizinisches Board.

Die spezifische Qualitätssicherung präsentiert sich in der Schweiz aktuell wie folgt: Der Bericht über ein öffentliches
Gesundheitsportal von eHealth Suisse (Koordinationsorgan eHealth Bund und Kantone) vom Januar 2012 misst der Qualitätssicherung von öffentlichen und privaten Gesundheitswebsites grosse Bedeutung bei. Nationalrätin Edith Graf-Litscher fragte den Bundesrat im März 2012 an, welche Wichtigkeit und Notwendigkeit er dem Qualitätssicherungslabel HON zuschreibt, damit die besonders sensiblen Gesundheitsinformationen auf Websites verlässlich sind. Der Bundesrat ant-

\section{Die Qualitätssicherung von \\ Gesundheitsinformationen ist zentral und muss darum gefördert werden.}

wortete darauf, dass bereits im Jahr 2009 der Steuerungsausschuss eHealth Suisse empfohlen hat, dass Bund und Kantone die Sensibilisierung für eine Qualitätssicherung von Gesundheitsinformationen und deren Umsetzung fördern sollten. Im Januar 2012 hatte der Steuerungsausschuss von eHealth Suisse seine Empfehlung erneuert und forderte die Anbieter von gesundheitsrelevanten Websites auf, ihre Internetangebote zertifizieren zu lassen, z.B. mit dem Label der HON-Stiftung.

Der HONcode ist das national und international am meisten verbreitete Qualitätslabel für Gesundheitsinformationen auf dem Web. In der Schweiz wurden bisher rund

310 Gesundheitswebsites mit dem Label zertifiziert; in Frankreich, wo die HON-Zertifizierung finanziell durch die Haute Autorité de la Santé (HAS) mitgetragen wird, sind es rund 1200 Websites.

Auf Bundesebene besteht gemäss geltendem Recht keine gesetzliche Grundlage, um Qualitätslabels für gesundheitsbezogene Internetseiten finanziell zu unterstützen. Um jedoch möglichst gute Rahmenbedingungen für die Umsetzung der Strategie eHealth Suisse zu schaffen, organisierte der Bund Ende Mai 2013 eine Geberkonferenz. Damit wollte der Bundesrat dazu beitragen, die finanziellen Grundlagen für die Arbeit der HON-Stiftung durch Bezuschussung von Privaten und Kantonen in den nächsten Jahren möglichst zu verbessern. Weil das Internet heutzutage eine wichtige Informationsquelle für Patienten darstellt, sind die Qualität von Gesundheitsinformationen im Internet und damit der HONCode langfristig zu sichern.

Dr. med. Gert Printzen, Mitglied des Zentralvorstandes der FMH, Verantwortlicher Ressort Medizinische Informatik und eHealth 